\title{
True Nature of an Archetypal Self-Assembly System: Mobile Au-Thiolate Species on Au(111)
}

\author{
Miao Yu, ${ }^{1}$ N. Bovet, ${ }^{2}$ Christopher J. Satterley, ${ }^{2}$ S. Bengió, ${ }^{1,3}$ Kevin R. J. Lovelock, ${ }^{2}$ P. K. Milligan, ${ }^{2}$ Robert G. Jones, ${ }^{2}$ \\ D. P. Woodruff, ${ }^{1, *}$ and V. Dhanak ${ }^{4}$ \\ ${ }^{1}$ Physics Department, University of Warwick, Coventry CV4 7AL, United Kingdom \\ ${ }^{2}$ Department of Physical Chemistry, School of Chemistry, University of Nottingham, Nottingham NG7 2RD, United Kingdom \\ ${ }^{3}$ INIFTA, CONICET-UNLP, Casilla de Coreo 16 Sucursal 4, 1900 La Plata, Argentina \\ ${ }^{4}$ Surface Science Research Centre, University of Liverpool and Daresbury Laboratory, Warrington WA4 4AD, United Kingdom
}

(Received 6 July 2006; published 20 October 2006)

\begin{abstract}
Alkanethiol self-assembled monolayer (SAM) phases on $\mathrm{Au}(111)$ have been assumed to involve direct $\mathrm{S}$ head group bonding to the substrate. Using X-ray standing wave experiments, we show the thiolate actually bonds to gold adatoms; self-organization in these archetypal SAM systems must therefore be governed by the movement of these Au-S- $R$ moieties on the surface between two distinct local hollow sites on the surface. The results of recent $a b$ initio total energy calculations provide strong support for this description, and a rationale for the implied significant molecular mobility in these systems.
\end{abstract}

DOI: $10.1103 /$ PhysRevLett.97.166102

PACS numbers: 68.43.Fg, 68.49.Uv, 81.16.Dn

Self-assembled monolayers (SAMs) of molecular systems have attracted huge interest over the last decade or more (e.g., [1-3]). The archetypal SAM system of straightchain alkanethiols $\left[\mathrm{CH}_{3}\left(\mathrm{CH}_{2}\right)_{n-1} \mathrm{SH}\right]$ chemisorbed on $\mathrm{Au}(111)$ is the subject of many investigations, yet the structure of the molecule-substrate interface, which strongly influences the molecular ordering, remains in doubt [4]. Here we show that a quantitative investigation of this interface structure leads us to conclude that the true nature of the self-assembly in these SAMs is not organization of adsorbed molecules on the $\mathrm{Au}(111)$ surface, but rather organization on the surface of Au-thiolate complexes formed by the bonding of the deprotonated thiol molecule to single Au adatoms. Our results resolve two major puzzles in these systems: (i) why do all previous total energy calculations fail to agree with experiment over the adsorption site of the simplest methylthiolate species ( $n=1, \mathrm{CH}_{3} \mathrm{~S}$ ) [5,6]; (ii) why is interchange between the several ordered structures of the molecules in their standing-up orientation so facile? This modified view of the interface has major implications for our understanding of the local ordering in these SAMs, and the influence of the corrugated adsorbate-substrate potential relative to the interadsorbate forces.

The dearth of experimental determinations of the local adsorption site of the $\mathrm{S}$ head group of the deprotonated thiol (thiolate) on the Au surface, has led to many theoretical total energy calculations. Most are for the simplest (methyl) thiolate in the saturation coverage $(\sqrt{3} \times$ $\sqrt{3}) R 30^{\circ}$ (hereafter $\sqrt{3}$ ) surface mesh in which, on symmetry grounds, all adsorbate species must have identical local geometries. These calculations have favored occupation of either threefold coordinated hollow sites or twofold coordinated bridging sites, or intermediate hollow-bridge sites [7-14]. By contrast, two wholly independent experimental structure determinations have shown the true ad- sorption site for methanethiolate on $\mathrm{Au}(111)$ is atop [5,6]. For the highest coverage "standing-up" phases of longer chain alkyl thiolates $(n>1)$, the $\sqrt{3}$ phase generally coexists (e.g., $[4,15])$ with a $(3 \times 2 \sqrt{3})$ rect. phase (hereafter $2 \sqrt{3}$ - often referred to as a $c(4 \times 2)$ supercell), having the same nominal coverage of 0.33 monolayer. On symmetry grounds, this larger unit mesh phase must contain two or more different local thiolate adsorption geometries. Facile interchange between the two phases has been reported, induced by small temperature changes or scanning with an STM tip $[4,16]$. Based on atomic-scale STM images, it has been suggested that there may be as many as 5 different local structural arrangements of the adsorbed thiolates corresponding to the same $2 \sqrt{3}$ periodicity [17].

Here we present the results of new structural measurements using normal incidence $\mathrm{x}$-ray standing waves (NIXSW) $[18,19]$ for hexylthiolate $(n=6)$ and octylthiolate $(n=8)$ SAMs on $\mathrm{Au}(111)$ in mixed $\sqrt{3}+2 \sqrt{3}$ phases, as well as tertiary-butylthiolate $\left[\left(\mathrm{CH}_{3}\right)_{3} \mathrm{CS}\right]$ in a complex $(\sqrt{19} \times \sqrt{19}) R 23.4^{\circ}$ phase (hereafter $\left.\sqrt{19}\right)$, and compare these with published data for both methylthiolate (see above) and $n$ butylthiolate $(n=4)$ in the $\sqrt{3}$ phase. We show that all the data are consistent with the $\mathrm{S}$ head group being atop a surface Au adatom which may occupy either of the two inequivalent threefold coordinated hollow sites on the underlying $\mathrm{Au}(111)$ substrate. These two sites lie directly above the second and third layer $\mathrm{Au}$ substrate atoms (Fig. 1) and correspond to local hexagonal-closepacked (hcp) and face- centered-cubic (fcc) stacking sequences that are known to have closely similar energies in fcc metals. The facile movement of this Au-thiolate complex between the fcc and hcp hollow sites, predicted in recent density-functional theory (DFT) total energy calculations [20], provides a simple mechanism for the interchange between the $\sqrt{3}$ phase and one or more $2 \sqrt{3}$ phases, 


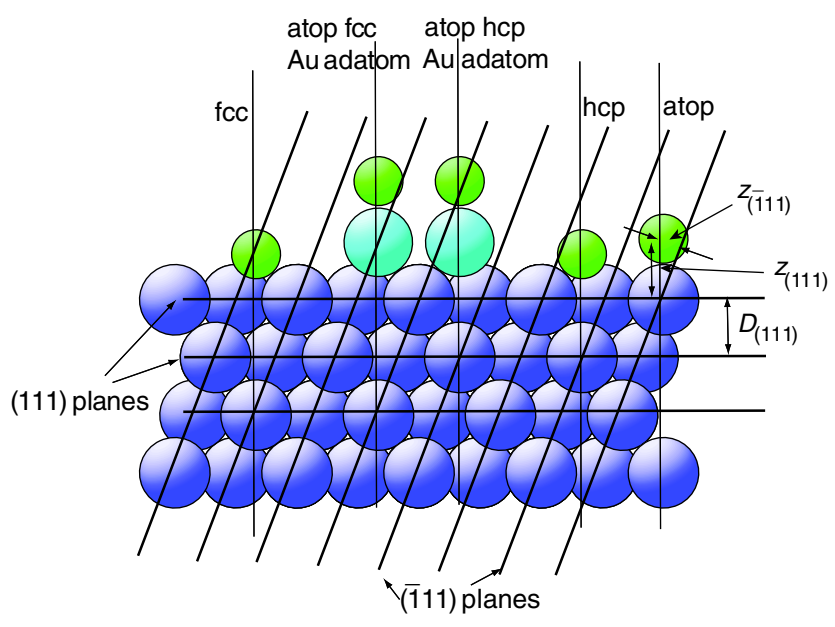

FIG. 1 (color online). Side view of $\mathrm{Au}(111)$ showing (111) and (111) scattering planes, the 3 high-symmetry adsorption sites for $\mathrm{S}$ (green) on an unreconstructed surface, and the $2 \mathrm{~S}$ adsorption sites atop Au adatoms (pale blue) in fcc and hcp hollows. A S-Au nearest-neighbor distance of $2.50 \AA$ has been used for all sites. $\mathrm{XSW}$ adsorbate triangulation distances are shown for the atop site.

and a basis for understanding the whole class of $\mathrm{Au} /$ thiolate SAMs.

The NIXSW experiments were conducted on beam line 4.2 [21] of the Synchrotron Radiation Source at Daresbury Laboratory. A concentric hemispherical electron energy analyzer was used to measure the intensity of the S $1 s$ and S KLL Auger electron emissions, used to monitor the $\mathrm{x}$-ray absorption at the $\mathrm{S}$ head group. The $\mathrm{Au}(111)$ crystal sample was cleaned in situ by argon ion bombardment and annealing cycles to produce a clean well-ordered $(22 \times \sqrt{3})$ rect. "herring-bone" reconstructed surface as assessed by the synchrotron radiation $\mathrm{x}$-ray photoelectron spectroscopy, Auger electron spectroscopy and LEED. The very high gas phase doses $\left(\sim 10^{-1}\right.$ mbar . s) needed to achieve the saturated standing-up phase of octanethiol were applied in the sample transfer chamber to avoid contamination of the UHV analysis chamber; the hexanethiol surface layers were prepared by immersion in an ethanolic solution of the thiol, followed by rinsing and drying in nitrogen gas prior to transfer to the UHV end station. The solution deposition method, in particular, is known to lead to the formation of coexistent $\sqrt{3}$ and $2 \sqrt{3}$ phase regions with the latter favored [22]. SAMs of tertiary butylthiolate were formed by exposure to a partial pressure of $\sim 10^{-7}$ mbar of ditertiary butyldisuphide.

NIXSW measurements were made at normal incidence to the (111) scatterer planes, parallel to the surface, to provide a measure of the sulfur head group adsorbate positions perpendicular to the surface, and at normal incidence to the (111) scatterer planes, to determine the lateral position of the sulfur atoms by triangulation, and hence the adsorption site (see Fig. 1). These NIXSW profiles were analyzed according to our standard procedures to extract the two associated structural parameters [18,19,23]: the coherent position $d_{H}$ ( $H$ specifies the scatterer plane Miller indices) and the coherent fraction $f_{\text {co }}$. If an absorber occupies a single well-defined site, $d_{H}$ is equal to the perpendicular distance of this site from the scattering planes, while $f_{\text {co }}$ is a measure of the degree of local order, a value of unity implying perfect order. Low values of $f_{\text {co }}$ much smaller than that for the substrate absorption typically imply two or more coexistent local adsorption geometries.

The NIXSW parameter values from these experiments, and our earlier measurements of the methylthiolate and $n$-butylthiolate species in the $\sqrt{3}$ phase, [6] are summarized in Table I. All five thiolates have closely similar $d_{(111)}$ values and associated $f_{(111)}$ values quite close to unity. This implies that all the $\mathrm{S}$ atoms are at essentially the same layer spacing from the underlying $\mathrm{Au}(111)$ surface, even though the complex phases must involve multiple local adsorption geometries relative to the substrate. The $\mathrm{x}$-ray standingwave technique (XSW) measures the adsorbate layer spacing relative to the nearest extended substrate scatterer plane, so the true distance to the nearest Au atoms may differ by an integral number of substrate interlayer spacings. Table I $d_{(111)}$ values have been increased by one interlayer spacing $D_{(111)}(2.36 \AA)$.

The local adsorption site may be obtained from the measured $d_{(-111)}$ values by simple geometrical triangulation. The three three-fold-symmetric adsorption sites on fcc (111) surfaces (atop, hcp hollow, fcc hollow) correspond to positions atop first, second, and third layer substrate atoms. If the true adsorbate-outermost substrate layer spacing is $z_{(111)}$, these three sites correspond to atop sites with effective layer spacings of $z_{(111)}, z_{(111)}+D_{(111)}$ and $z_{(111)}+2 D_{(111)}$ (Fig. 1). The (111) planes are inclined at an angle of $70.5^{\circ}$ to the (111) surface planes, so these sites are spaced above the $(\overline{1} 11)$ planes by $z_{(111)} / 3,\left(z_{(111)}+\right.$ $\left.D_{(111)}\right) / 3$ and $\left(z_{(111)}+2 D_{(111)}\right) / 3$ (the factor $1 / 3$ being $\left.\cos 70.5^{\circ}\right)$. Equating $d_{(111)}$ to $z_{(111)}$, Table I shows the predicted $d_{(-111)}$ values for atop, fcc hollow, and hcp hollow sites in the final columns. Bridge site occupation leads to a $d_{(-111)}$ value midway between those for the two hollow sites, but has a $f_{(-111)}$ value reduced by a factor of 3 $[18,19]$. All other possible lower symmetry sites lead to one of these four $d_{(-111)}$ values, but with lower $f_{(-111)}$ values $[18,19]$.

The agreement between the experimental values and those predicted for the atop site is essentially perfect for the methylthiolate and $n$-butylthiolate in the $\sqrt{3}$ phase, in which all sites must be identical [6]. For the mixed phase hexylthiolate and octylthiolate layers, and the complexphase $t$-butylthiolate, all of which must involve two or more distinct adsorption sites, none of the predicted single-site triangulation values agree with experiment. The experimental $d_{(-111)}$ values for these systems lie between those predicted for atop and fcc hollows, so accept- 
TABLE I. Summary of the NIXSW fitting parameters obtained for the standing-up saturation-coverage phases formed by a series of alkanethiols on $\mathrm{Au}(111)$. Numbers in parentheses are estimated errors in the least significant figure; "mixed" indicates a mixture of $\sqrt{3}$ and $2 \sqrt{3}$ phases.

\begin{tabular}{lccccccc}
\hline \hline Adsorbate/phase & $d_{(111)}(\AA)$ & $f_{(111)}$ & $d_{(-111)}(\AA)$ & $f_{(-111)}$ & \multicolumn{3}{c}{ Calculated $d_{(-111)}(\AA)$} \\
\hline & & & & & atop & fcc & hcp \\
$C_{1} / \sqrt{3}[6]$ & $2.48(5)$ & $0.83(5)$ & $0.86(5)$ & $0.65(5)$ & 0.83 & 0.04 & 1.61 \\
$C_{4} / \sqrt{3}[6]$ & $2.47(5)$ & $0.77(5)$ & $0.84(5)$ & $0.56(7)$ & 0.82 & 0.04 & 1.61 \\
$C_{6} /$ mixed & $2.50(5)$ & $0.82(7)$ & $0.48(5)$ & $0.62(9)$ & 0.83 & 0.05 & 1.62 \\
$C_{8} /$ mixed & $2.55(5)$ & $0.79(5)$ & $0.59(5)$ & $0.73(7)$ & 0.85 & 0.06 & 1.63 \\
$t-C_{4} / \sqrt{19}$ & $2.48(5)$ & $0.86(6)$ & $0.67(8)$ & $0.50(7)$ & 0.83 & 0.04 & 1.61 \\
\hline \hline
\end{tabular}

able models must involve a mixture of these two sites at the same layer spacing from the underlying substrate. At first sight this requirement seems impossible to satisfy. Figure 1 shows clearly that direct bonding of the sulfur atom in a mixture of atop and hollow sites leads to a distribution of $z$ values that is inconsistent with the experimental measurements unless there are unreasonably large differences in $\mathrm{Au}-\mathrm{S}$ bond lengths in the two sites. Consider, however, the consequences of thiolate adsorption atop an Au adatom on the $\mathrm{Au}(111)$ surface. If this $\mathrm{Au}$ adatom occupies an fcc hollow site, the XSW triangulation will be the same as for an atop site, because it is atop an Au atom in a bulkcontinuation site of the underlying solid. If the Au adatom occupies an hcp hollow site, the thiolate $\mathrm{S}$ atom is then $D_{(111)}+z_{(111)}$ above the hcp site, equivalent to being $z_{(111)}$ above an fcc hollow site with respect to the underlying bulk (Fig. 1). This second S adsorption geometry is the only one which satisfies the constraints of the (111) and (111) NIXSW data, and the requirement that the two inequivalent thiolate sites should have closely similar Au-S bond lengths.

Our data can therefore only be interpreted consistently in terms of Au-S- $R$ moieties on the surface (where $R$ denotes the rest of the thiolate species), and it is the location of these moieties that defines the structural phase; in the $\sqrt{3}$ phase the Au adatoms are all in the fcc sites, but in the $2 \sqrt{3}$ phase $(s)$ a mixture of fcc and hcp sites is occupied. The $2 \sqrt{3}$ phase contains four thiolate species per primitive unit mesh, so models are possible with 1,2 , or 3 thiolate $\mathrm{S}$ head group atoms atop hcp Au adatoms with the remainder atop fcc Au adatoms. Figure 2 illustrates two possible ways of ordering the adatoms, having 3:1 and 2:2 ratios of fcc:hcp Au adatom site occupations. The $\sqrt{19}$ phase of the $t$-butylthiolate also involves a mixture of these same two local geometries, the larger $d_{(-111)}$ value being consistent with more than $50 \%$ of the Au adatoms occupying fcc hollows. Note that the models of Fig. 2 imply S-S distances as low as $3.33 \AA$, much shorter than the $4.99 \AA$ of the $\sqrt{3}$ phase, but much longer than that of a disulphide $(2.04 \AA)$; the NIXSW results would also be consistent with $\mathrm{S}$ off atop the Au adatoms by $0.2-0.3 \AA$, allowing further increase of these S-S distances.

This model provides a solution to both the puzzles raised in our introduction. First, it provides a rationale for the failure of all of the many ab initio total energy calculations to correctly identify the atop adsorption site for methylthiolate. All but one of these calculations failed to considered the possibility of the Au adatom model. Molina and Hammer [13] did consider this "inverse honeycomb model" for which the atop site had the lowest energy, but identified an alternative ("honeycomb") reconstruction model as giving a lower total energy. Another recent DFT calculation [20] has found that ethylthiolate adsorbed atop an Au adatom is energetically favorable, not only in isolation, but also in a $\sqrt{3}$ phase; moreover, the energy of the thiolate-Au adatom complex is found to be almost identical in the fcc and hep sites (the calculations actually favor the hcp site) and the barrier to diffusion between the two sites is extremely low. Our finding that the hexylthiolate and octylthiolate layers can be reconciled with partial occupation of the two adatom sites is thus clearly consistent with this view, while the low barrier to diffusion between the sites coupled with the idea that the $2 \sqrt{3}$ phase

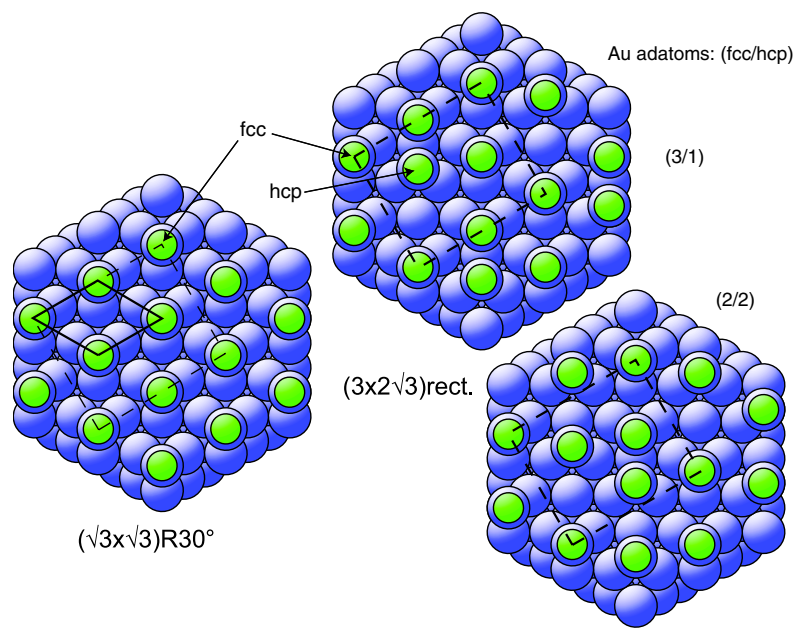

FIG. 2 (color online). Schematic plan view of (left) $\sqrt{3}$ phase of alkane thiolate on $\mathrm{Au}(111)$ showing the $\mathrm{S}$ head group atom (green) atop Au adatoms (pale blue) in fcc hollow sites relative to the underlying surface; (right) two possible $2 \sqrt{3}$ phase models with $\mathrm{S}$ head group atoms atop Au adatoms, with 3:1 and 2:2 ratios of fcc and hep site occupations. The alkane chain atoms are omitted for clarity. The $\sqrt{3}$ and $2 \sqrt{3}$ unit meshes are shown by full and dashed lines, respectively. 
involves co-occupation of both sites provides a clear rationale for the observed facile switching between the $\sqrt{3}$ phase and $2 \sqrt{3}$ phases.

There are a few literature reports which appear to conflict with our interpretation. STM images of $2 \sqrt{3}$ phases have typically been interpreted qualitatively in terms of mixed molecular conformations with all $\mathrm{S}$ head group atoms in hollow sites [17] or in mixed hollow and bridge sites [24]; these S sites are clearly incompatible with our data. For methylthiolate, the photoelectron diffraction study identifying atop site adsorption [5] reported better agreement with an unreconstructed $\mathrm{Au}(111)$ surface than an Au adatom model, but this conclusion may be sensitive to the choice of surface vibrational parameters in the modeling of the photoelectron diffraction. Moreover, a surface $x$-ray diffraction study of the $2 \sqrt{3}$ phase of hexanedecylthiolate $(n=16)$ on $\mathrm{Au}(111)$ [15] concluded that the thiolate occupies the fcc and hcp hollow sites of an unreconstructed surface, although the same group concludes that for the $\sqrt{3}$ phase the atop site is favored by the diffraction data [25]. In neither case, however, was the $\mathrm{Au}$ adatom model tested. Interestingly, one earlier NIXSW study of the $2 \sqrt{3}$ phase of decylthiolate $(n=10)$ on $\mathrm{Au}(111)$ [26] found (111) and (111) coherent position values $(0.23$ and $0.59 \AA)$ very similar to those of our measurements for octylthiolate and hexylthiolate. These data may therefore also be interpreted in terms of our $\mathrm{Au}$ adatom model.

Very recently, low temperature STM studies of the early stages of thiolate SAM formation on $\mathrm{Au}(111)$ [27] have provided direct evidence of the extraction of surface $\mathrm{Au}$ atoms to form $\mathrm{Au}$ adatom-thiolate moieties; this microscopic evidence provides strong support for our model of the ordered SAM phases.

The fact that alkylthiolate SAMs on Au(111) involve Au-thiolate moieties, rather than thiolates bonded directly to the atomically flat substrate, will require major reconsideration of the whole basis of the self-organization, which is determined by the relative strength of the intermolecular interactions and the lateral corrugation of the substrate potential. In particular, the corrugation of importance is not that associated with the thiolate bonding to different sites, but rather the Au-thiolate bonding in different sites. In the $\sqrt{3}$ phase, with only one adsorbate molecule per primitive unit mesh, the substrate potential corrugation must dominate the ordering. In the $2 \sqrt{3}$ phase, the Au-thiolate complex occupies both fcc and hcp sites, the energy of these local sites is essentially identical, and the barrier for movement between them is small; intermolecular interactions then can then play a far greater role in the self-organization. The fact that the formation of $\mathrm{Au}$ thiolate surface complexes accompanies the Au/thiol surface reaction must also influence the reaction dynamics, and is clearly consistent with the recent finding that the deprotonation reaction occurs at defects sites on the sur- face [28]. Finally, our observation of the same behavior in a completely different complex phase of tertiarybutlythiolate on $\mathrm{Au}(111)$ (for which steric hindrance precludes the formation of the $\sqrt{3}$ phase), suggests the adatom-thiolate moiety may also occur in the far wider class of (functionalized) thiolate SAMs on $\mathrm{Au}(111)$.

The authors acknowledge the allocation of SRS beam time by the Council for the Central Laboratories for the Research Councils (CCLRC). M. Y. acknowledges the support of the Warwick Postgraduate Research program and an ORS grant and N.B., C. S., and K. L. acknowledge the support of the University of Nottingham and the EPSRC for studentships.

*Corresponding author.

Electronic address: d.p.woodruff@warwick.ac.uk

[1] L. H. Dubois and R. G. Nuzzo, Annu. Rev. Phys. Chem. 43, 437 (1992).

[2] A. Ulman, Chem. Rev. 96, 1533 (1996).

[3] F. Schreiber, J. Phys. Condens. Matter 16, R881 (2004).

[4] C. Vericat, M.E. Vela, and R. C. Salvarezza, Phys. Chem. Chem. Phys. 7, 3258 (2005).

[5] H. Kondoh, Phys. Rev. Lett. 90, 066102 (2003).

[6] M. G. Roper et al., Chem. Phys. Lett. 389, 87 (2004).

[7] H. Sellers et al., J. Am. Chem. Soc. 115, 9389 (1993).

[8] H. Grönbeck, A. Curioni, and W. Andreoni, J. Am. Chem. Soc. 122, 3839 (2000).

[9] Y. Yourdshahyan, H. K. Zhang, and A. M. Rappe, Phys. Rev. B 63, 081405 (2001).

[10] T. Hayashi, Y. Morikawa, and H. Nozoye, J. Chem. Phys. 114, 7615 (2001).

[11] M. C. Vargas et al., J. Phys. Chem. B 105, 9509 (2001).

[12] J. Gottschalck and B. Hammer, J. Chem. Phys. 116, 784 (2002).

[13] L. M. Molina and B. Hammer, Chem. Phys. Lett. 360, 264 (2002).

[14] Y. Morikawa et al., Surf. Sci. 507-510, 46 (2002).

[15] X. Torrelles et al., Langmuir 20, 9396 (2004).

[16] F. Terán Arce et al., J. Chem. Phys. 109, 5703 (1998).

[17] B. Lüssem et al., Langmuir 21, 5256 (2005).

[18] D. P. Woodruff, Prog. Surf. Sci. 57, 1 (1998).

[19] D. P. Woodruff, Rep. Prog. Phys. 68, 743 (2005).

[20] F. P. Cometto et al., J. Phys. Chem. B 109, 21737 (2005).

[21] A. W. Robinson et al., Rev. Sci. Instrum. 66, 1762 (1995).

[22] C. Vericat, M. E. Vela, and R. C. Salvarezza, Phys. Chem. Chem. Phys. 7, 3258 (2005).

[23] The Igor routines of XSWFIT can be obtained from robert.g.jones@nottingham.ac.uk.

[24] J. Zhang, Q. Chi, and J. Ulstrup, Langmuir 22, 6203 (2006).

[25] X. Torelles et al., J. Phys. Chem. B 110, 5586 (2006).

[26] P. Fentner et al., Surf. Sci. 412-413, 213 (1998).

[27] P. Maksymovych, D. C. Sorescu, and J. T. Yates, Jr., Phys. Rev. Lett. 97, 146103 (2006).

[28] I. I. Rzeznica, J. Lee, P. Maksymovych, and J. T. Yates, Jr., J. Phys. Chem. B 109, 15992 (2005). 